\title{
Electroweak baryogenesis via bottom transport
}

\author{
Tanmoy Modak ${ }^{1, *}$ and Eibun Senaha ${ }^{2, \dagger}$ \\ ${ }^{1}$ Department of Physics, National Taiwan University, Taipei 10617, Taiwan \\ ${ }^{2}$ Center for Theoretical Physics of the Universe, Institute for Basic Science (IBS), Daejeon 34126, Korea
}

(Received 21 January 2019; published 18 June 2019)

\begin{abstract}
We consider a scenario in which an extra bottom Yukawa coupling can drive electroweak baryogenesis in the general two-Higgs doublet model. It is found that the new bottom Yukawa coupling with $\mathcal{O}(0.1)$ in magnitude can generate the sufficient baryon asymmetry without conflicting existing data. We point out that future measurements of the bottom Yukawa coupling at High-Luminosity Large Hadron Collider and International Linear Collider, together with the $C P$ asymmetry of $B \rightarrow X_{s} \gamma$ at SuperKEKB provide exquisite probes for this scenario.
\end{abstract}

DOI: $10.1103 /$ PhysRevD.99.115022

\section{INTRODUCTION}

The existence of the baryon asymmetry of the Universe (BAU) is firmly established by various cosmological observations such as the cosmic microwave background and big-bang nucleosynthesis [1]. However, its origin is still unclear, which motivates one to search for physics beyond the Standard Model (SM).

A plethora of baryogenesis scenarios have been proposed so far. After the discovery of the Higgs boson at Large Hadron Collider (LHC) in 2012 [2], significant attention has been paid in particular to electroweak baryogenesis (EWBG) [3,4] for its close connection to Higgs physics. One of the necessary conditions for the successful EWBG is that electroweak phase transition (EWPT) is strongly first order, which requires an extra particle with a mass of sub-TeV that couples to the Higgs boson. Wellstudied examples are new scalar or vector particles that modify the Higgs potential by tree-level mixings and/or loop effects. In addition to these conventional cases, it has been pointed out that even fermions could induce such an effect if they couple to the Higgs boson strongly [5] (for a recent study, see Ref. [6]). But in this case, additional bosonic degrees of freedom are needed to evade vacuum instability.

Furthermore, $C P$ violation relevant to EWBG often arises from Higgs-Yukawa interactions. Therefore, the

\footnotetext{
*tanmoyy@hep1.phys.ntu.edu.tw

‘senaha@ibs.re.kr
}

Published by the American Physical Society under the terms of the Creative Commons Attribution 4.0 International license. Further distribution of this work must maintain attribution to the author(s) and the published article's title, journal citation, and DOI. Funded by SCOAP ${ }^{3}$.
Higgs signal strengths are inevitably modified by the new physics (NP) effects.

Recently, the Higgs boson decay to bottom quarks has been observed at the LHC. Its signal strength relative to the SM expectation is $1.01 \pm 0.12$ (stat. $)_{-0.15}^{+0.16}$ (syst.) at ATLAS [7] and $\mu=1.04 \pm 0.14$ (stat.) \pm 0.14 (syst.) at CMS [8], respectively. While the measured values are consistent with the SM, however, there still exists sufficient room for NP.

The NP effects in the bottom sector are of great importance for $B$ physics as well. In addition to the ongoing LHCb experiment, Belle-II at KEK will start collecting data (phase 3) in early 2019 and accumulate it up to $50 \mathrm{ab}^{-1}$ by 2024. One of the goals is to search for $C P$ violation beyond the Cabibbo-Kobayashi-Maskawa (CKM) framework [9]. It is of broad interest whether such a $C P$ violation can be related to the BAU.

In this paper, we consider a scenario in which additional bottom Yukawa coupling is responsible for the BAU and discuss its implications to collider phenomenology as well as $B$ physics, especially $B \rightarrow X_{s} \gamma$. We take the general two Higgs double model (G2HDM) [10] as a benchmark model. For previous studies of EWBG in the model, see, e.g., Refs. [11-17]. For instance, in Ref. [16] a scenario in which BAU is sourced by new $C P$ violation in the up-type Yukawa couplings is considered. This EWBG scenario is very efficient as long as an extra top Yukawa coupling is complex and $\mathcal{O}(0.1-1)$ in magnitude. In such a case, there is no strong motivation to consider additional $C P$ violations in the down-type Yukawa couplings. In the current analysis, however, we explore the EWBG possibility assuming that the up-type Yukawa couplings do not provide any new $C P$ violations. Therefore, the current analysis is complementary to the above top-driven scenario. While it is also discussed that the $C P$ violation required to generate BAU may also come from a flavor-changing bottom Yukawa coupling [14] and evade the constraint from electric dipole 
moments (EDMs) of the electron; however, it is a wellknown fact that the flavor-diagonal Yukawa couplings are much more efficient $[12,13,15,16]$. Therefore, such possibilities should also be clarified in the bottom transport scenario. In contrast to the claim of Ref. [14], we cannot find any successful EWBG regions utilizing the flavor-changing bottom Yukawa coupling in our numerical analysis.

We point out that the extra flavor-diagonal bottom Yukawa coupling of $\mathcal{O}(0.1)$ in magnitude can offer the successful EWBG without upsetting existing experimental constraints. It is found that, except for some corner of the parameter space, most EWBG-viable regions can fully be covered by Higgs signal strength measurements at HighLuminosity LHC (HL-LHC) and future colliders such as International Linear Collider (ILC). Besides, such a scenario can also be tested by $B$ physics observables, especially the branching ratio and $C P$ asymmetry of $B \rightarrow X_{s} \gamma$ at SuperKEKB.

The paper is organized as follows. In Sec. II we discuss the formalism for EWBG via bottom transport. Section III is dedicated to the experimental constraints on the parameter space, which is relevant for baryogenesis. The results are summarized in Sec. IV, with some discussions and the conclusion in Sec. V.

\section{BAU VIA BOTTOM TRANSPORT}

The Yukawa interactions of the G2HDM in a generic basis are parametrized as

$$
-\mathcal{L}_{Y}=\bar{f}_{L}\left(Y_{1} \Phi_{1}+Y_{2} \Phi_{2}\right) f_{R}+\text { H.c. },
$$

where $\Phi_{1,2}$ are the Higgs doublets whose vacuum expectation values (VEVs) are parametrized as $v_{1}$ and $v_{2}$, respectively, and $f=u, d, e$. In the mass eigenbasis of the fermions and Higgs bosons, one has

$$
-\mathcal{L}_{Y} \ni \bar{f}_{L} y_{\phi}^{f} f_{R} \phi+\bar{u}\left[V \rho^{d} P_{R}-\rho^{u \dagger} V P_{L}\right] d H^{+}+\text {H.c. },
$$

where $P_{L, R}=\left(1 \mp \gamma_{5}\right) / 2, V$ is the CKM matrix, $H^{+}$is the charged scalar and $\phi=h, H, A$, with $h$ identified as $125 \mathrm{GeV}$ boson, and $H$ and $A$ are $C P$-even and $C P$-odd scalars, respectively. $y_{\phi}^{f}$ are the $3 \times 3$ matrices defined, respectively, as

$$
\begin{gathered}
y_{h i j}^{f}=\frac{\lambda_{i}^{f}}{\sqrt{2}} \delta_{i j} s_{\beta-\alpha}+\frac{\rho_{i j}^{f}}{\sqrt{2}} c_{\beta-\alpha}, \\
y_{H i j}^{f}=\frac{\lambda_{i}^{f}}{\sqrt{2}} \delta_{i j} c_{\beta-\alpha}-\frac{\rho_{i j}^{f}}{\sqrt{2}} s_{\beta-\alpha}, \\
y_{A i j}^{f}=\mp \frac{i \rho_{i j}^{f}}{\sqrt{2}},
\end{gathered}
$$

where $i, j$ are flavor indices, $\lambda_{i}^{f}=\sqrt{2} m_{i}^{f} / v\left(v=\sqrt{v_{1}^{2}+v_{2}^{2}}=\right.$ $246 \mathrm{GeV}), s_{\beta-\alpha}=\sin (\beta-\alpha)$, and $c_{\beta-\alpha}=\cos (\beta-\alpha)$ with $\alpha$ being the mixing angle between $h$ and $H$ while $\beta=\tan ^{-1}\left(v_{2} / v_{1}\right)$. The negative (positive) sign in Eq. (5) is for the up (down)-type fermions. The $3 \times 3$ matrices $\rho^{f}$ are in general complex and can break $C P$ explicitly and/or induce the flavor-changing processes. Note that the Yukawa coupling for $h$ is reduced to the SM in the limit of $c_{\beta-\alpha} \rightarrow 0$ (alignment limit). In the current study, we consider the case in which $\rho_{t t}^{u}, \rho_{b b}^{d}$, and $\rho_{e e}^{e}$ are nonzero and set all other $\rho_{i j}=0$ for simplicity. Furthermore, $\rho_{t t}^{u}$ is assumed to be real (for a complex $\rho_{t t}$ case, see Ref. [16]). As discussed below, the nonzero $\rho_{e e}^{e}$ plays a pivotal role in realizing a cancellation mechanism in EDM of the electron [16]. Hereafter, we omit the superscripts of $\rho$ 's for notational simplicity.

As demonstrated in Refs. $[13,16]$, with a specific ansatz for $Y_{1,2}, \rho_{b b}$ is given by ${ }^{1}$

$$
\operatorname{Im}\left(\rho_{b b}\right)=-\frac{1}{\lambda_{b}} \operatorname{Im}\left[\left(Y_{1}\right)_{b s}\left(Y_{2}\right)_{b s}^{*}\right] .
$$

Therefore, $\rho_{b b}$ is correlated with the $b-s$ changing interactions in the symmetric phase, where the Higgs VEVs are zero. This correlation is also confirmed in a basis-invariant manner in Ref. [13]. Since we consider the VEVs as the small perturbation in calculating the BAU (VEV insertion approximation [18]), the $C P$-violating source term arising from the $b-s$ transitions takes the form

$$
S_{\mathrm{CPV}}=C_{\mathrm{BAU}} \operatorname{Im}\left[\left(Y_{1}\right)_{b s}\left(Y_{2}\right)_{b s}^{*}\right],
$$

where $C_{\mathrm{BAU}}$ denotes a dynamical factor for the scattering processes among the bottom/strange quarks and bubble wall (for the explicit form, see Refs. [15,16]). While this baryogenesis mechanism is the same as in Ref. [14], the correlation of Eq. (6) is unclear in [14], leading to different phenomenological consequences. More explicitly, the BAU-related $C P$ violation seems correlated with $\rho_{b s}$ rather than $\rho_{b b}$ so that there are no severe EDM constraints, which is in stark contrast to our case and other work $[12,13,15,16]$. In principle, $\rho_{b s}$ EWBG could be possible as is the case of $\rho_{t c}$ EWBG discussed in Ref. [16]. To this end, however, $\rho_{b s}$ has to be $\mathcal{O}(1)$ in magnitude, which is not allowed experimentally.

We calculate the BAU using closed-time-path formalism applied in Refs. [13-16,18,19]. ${ }^{2}$ The relevant particle

\footnotetext{
${ }^{1}$ Since the exemplified Yukawa ansatz leads to massless strange quark, we do not use it in our numerical calculation and take more realistic Yukawa ansatz.

${ }^{2}$ While a lot of efforts have been made in developing the BAU calculation using closed-time-path formalism [13-22], there still exist theoretical challenges that prevent one from obtaining the robust value (for a review, see, e.g., Ref. [4]).Theoretical uncertainties are addressed when interpreting our results.
} 
number densities in our scenario are $\left\{Q_{3}=n_{t_{L}}+n_{b_{L}}\right.$, $\left.T=n_{t_{R}}, B=n_{b_{R}}, S=n_{s_{R}}, H=n_{H_{1}^{+}}+n_{H_{1}^{0}}+n_{H_{2}^{+}}+n_{H_{2}^{0}}\right\}$, which are expanded to the leading order in the chemical potential $\mu$ as $n_{b, f}=T^{2} \mu k_{b, f} / 6$, with $b(f)$ being bosons (fermions). One finds that $k_{b(f)}=2(1)$ in the massless limit. The coupled diffusion equations for those number densities in the plasma frame are given by

$$
\begin{gathered}
\partial_{\mu} j_{Q_{3}}^{\mu}=-\Gamma_{Y_{t}}\left(\xi_{Q_{3}}+\xi_{H}-\xi_{T}\right)-\Gamma_{M_{t}}^{-}\left(\xi_{Q_{3}}-\xi_{T}\right) \\
-2 \Gamma_{s s} N_{5}+S_{b_{L}}, \\
\partial_{\mu} j_{T}^{\mu}=\Gamma_{Y_{t}}\left(\xi_{Q_{3}}+\xi_{H}-\xi_{T}\right)+\Gamma_{M_{t}}^{-}\left(\xi_{Q_{3}}-\xi_{T}\right)+\Gamma_{s s} N_{5} \\
\partial_{\mu} j_{H}^{\mu}=-\Gamma_{Y_{t}}\left(\xi_{Q_{3}}+\xi_{H}-\xi_{T}\right)+\Gamma_{Y_{b s}}\left(\xi_{Q_{3}}-\xi_{H}-\xi_{S}\right)-\Gamma_{H} \xi_{H},
\end{gathered}
$$

$$
\begin{gathered}
\partial_{\mu} j_{B}^{\mu}=\Gamma_{s s} N_{5}, \\
\partial_{\mu} j_{S}^{\mu}=\Gamma_{s s} N_{5}-S_{b_{L}},
\end{gathered}
$$

where $\xi_{i}=n_{i} / k_{i}, N_{5}=2 \xi_{Q}-\xi_{T}-\xi_{S}-8 \xi_{B}$, and $\partial_{\mu} j_{i}^{\mu}=$ $\dot{n}_{i}-D_{i} \nabla^{2} n_{i}$ with $D_{i}$ denoting a diffusion constant. $S_{b_{L}}$ denotes the $C P$-violating source term induced by $\left(Y_{1,2}\right)_{b s}$ while $\Gamma_{Y_{t}}, \Gamma_{M_{t}}^{-}, \Gamma_{H}$, and $\Gamma_{s s}$ are the rates by top-Higgs interactions, top-bubble wall interactions, Higgs numberviolating interactions, and strong sphaleron [23], respectively. Since $\Gamma_{Y_{t}}, \Gamma_{\mathrm{ss}} \gg \Gamma_{M_{t}}^{-}$, the above coupled equations can be reduced to a single differential equation for $H$ [14,22,24]: $\dot{H}-\bar{D} \nabla^{2} H+\bar{\Gamma} H-\bar{S}+\mathcal{O}\left(1 / \Gamma_{s s}, 1 / \Gamma_{Y_{t}}\right)=0$, where $\bar{S}=k_{H}\left(k_{Q_{3}}-7 k_{T}+k_{B}\right) S_{b_{L}} /(a+b)$ with $a=k_{H}\left(9 k_{Q_{3}}+\right.$ $9 k_{T}+k_{B}$ ) and $b=9 k_{Q_{3}} k_{T}+k_{Q_{3}} k_{B}+4 k_{T} k_{B}$ (for $\bar{D}$ and $\bar{\Gamma}$, see Ref. [14]). After transforming from the plasma frame to the wall rest frame $\left(z \rightarrow \bar{z}=z+v_{w} t\right.$ with $v_{w}$ being the bubble wall velocity), $S_{b_{L}}(\bar{z}) \propto v_{w} \Delta \beta / L_{w}$, where $\Delta \beta$ is a variation of $\beta$ during the EWPT and $L_{w}$ the bubble wall width.

One can find the total left-handed number density as

$$
n_{L}(\bar{z}) \simeq \frac{r_{2} v_{w}^{2}}{\Gamma_{s s} \bar{D}}\left(1-\frac{D_{q}}{\bar{D}}\right) H(\bar{z})+\mathcal{O}\left(1 / \Gamma_{Y}\right)
$$

where $r_{2}=k_{H} k_{B}^{2}\left(5 k_{Q_{3}}+4 k_{T}\right)\left(k_{Q_{3}}+2 k_{T}\right) / a^{2}$ and $D_{q}$ is the quark diffusion constant. Assuming that $\bar{\Gamma}(\bar{z})$ is nonzero and constant for $\bar{z}>0$, one gets $H(\bar{z}) \simeq$ $e^{v_{w} \bar{z} / \bar{D}} k_{H} L_{w} S_{b_{L}} \sqrt{a} / \sqrt{\left(\Gamma_{M_{t}}^{-}+\Gamma_{H}\right)\left(k_{H}(a+b) \bar{D}\right)}$, where we also take the limits of $4 \bar{D} \bar{\Gamma} \gg v_{w}^{2}$ and $L_{w} \sqrt{\bar{\Gamma} / \bar{D}} \ll 1$. To leading order in our calculation, the $L_{w}$ dependence in the $H(\bar{z})$ dependence drops out since $S_{b_{L}} \propto 1 / L_{w}$.

After solving a diffusion equation for the baryon number density $\left(n_{B}\right)[19,20,25]$, one finds

$$
n_{B}=\frac{-3 \Gamma_{B}^{(\mathrm{sym})}}{2 D_{q} \lambda_{+}} \int_{-\infty}^{0} d z^{\prime} n_{L}\left(z^{\prime}\right) e^{-\lambda_{-} z^{\prime}}
$$

with $\lambda_{ \pm}=\left[v_{w} \pm \sqrt{v_{w}^{2}+4 \mathcal{R} D_{q}}\right] / 2 D_{q}, \quad \Gamma_{B}^{(\text {sym })}$ is the $B$ changing rate via sphaleron in the symmetric phase, and $\mathcal{R}=15 \Gamma_{B}^{(\mathrm{sym})} / 4$.

One comment on an approximation adopted in Ref. [14] is that the $C P$-conserving source term induced by $\left(Y_{1,2}\right)_{b s}$ is treated as the next-to-leading order due to the fact that it is smaller than the corresponding term induced by the top quark, and thus neglected. However, naively, the numerical impact of such a term may not be negligibly small. If so, the BAU based on Ref. [14] would be overestimated. In our numerical analysis, we regard the dropped term as the part of the theoretical uncertainties and defer the improvement of the BAU calculation to future work.

Note that EWBG becomes ineffective if $v_{w}$ approaches zero or gets bigger than about the speed of sound in the plasma $(1 / \sqrt{3} \simeq 0.58)$. In Refs. [26,27], however, it is found that $0.1 \lesssim v_{w} \lesssim 0.6$ in the softly $Z_{2}$ broken 2 HDMs (two Higgs doublet models), where the stronger EWPT corresponds to larger $v_{w}$. Since there is no serious study on $v_{w}$ in the G2HDM, we take $v_{w}=0.4$ as a reference value. For a numerical estimate of $n_{B}$, we take $D_{q}=8.9 / T$, $\Gamma_{B}^{(\text {sym })}=5.4 \times 10^{-6} T$, and $\Gamma_{s s}=3.2 \times 10^{-3} T$ with $T$ being the temperature.

We find the BAU-viable regions by requiring that $Y_{B}=$ $n_{B} / s$ should be greater than the observed value $Y_{B}^{\text {obs }}=$ $8.59 \times 10^{-11}$ [28], where $s$ denotes the entropy density.

The BAU can survive after the EWPT if the $B$-changing process is sufficiently suppressed. The rough criterion of the $B$ preservation is given as $v_{C} / T_{C} \gtrsim 1$, where $T_{C}$ denotes a critical temperature and $v_{C}$ is the Higgs VEV at $T_{C}$. In our numerical analysis, we calculate $v_{C} / T_{C}$ using a finite-temperature one-loop effective potential with thermal resummation.

\section{EXPERIMENTAL CONSTRAINTS}

Before showing the numerical results, we first outline the experimental constraints relevant to our study. The $\rho_{b b}$ coupling is constrained by several existing measurements such as Higgs signal strengths, the branching ratio of $B \rightarrow X_{s} \gamma\left[\mathcal{B}\left(B \rightarrow X_{s} \gamma\right)\right], \mathrm{EDM}$, and the asymmetry of the $C P$ asymmetry between charged and neutral $\mathcal{B}\left(B \rightarrow X_{s} \gamma\right)$ decay $\left(\Delta \mathcal{A}_{\mathrm{CP}}\right)$.

First we consider constraints from Higgs signal strength measurements. The presence of nonzero $c_{\beta-\alpha}$ and $\rho_{i j}$ modify the $h$ boson couplings $y_{h f f}$, as can be seen from Eq. (3). As a result $\rho_{b b}$ receives a stringent constraint if $c_{\beta-\alpha}$ is nonzero. For our analysis we incorporate the Run-2 combined measurements of Higgs boson couplings by CMS [29]. The result is based on $\sqrt{s}=13 \mathrm{TeV} p p$ collision with $35.9 \mathrm{fb}^{-1}$ (2016 data) and summarizes different signal 
strengths $\mu_{i}^{f}$ for a specific decay mode $i \rightarrow h \rightarrow f$. The signal strength $\mu_{i}^{f}$ is defined as

$$
\mu_{i}^{f}=\frac{\sigma_{i} \mathcal{B}^{f}}{\left(\sigma_{i}\right)_{\mathrm{SM}}\left(\mathcal{B}^{f}\right)_{\mathrm{SM}}}=\mu_{i} \mu^{f},
$$

where $\sigma_{i}$ is the production cross section for $i \rightarrow h$ and $\mathcal{B}^{f}$ is the branching ratio for $h \rightarrow f$, with $i=g g F$, $V B F, Z h, W h, t t h$ and $f=\gamma \gamma, Z Z, W W, \tau \tau, b b, \mu \mu$. We follow Refs. [10,30-32] for the expressions of different $\mu_{i}^{f}$. In particular, we take two production modes, gluon fusion $(g g F)$ and vector boson fusion $(V B F)$ in our analysis. We find that for the $g g F$ category, the sensitive decay modes are $\mu_{g g F}^{\gamma \gamma}, \mu_{g g F}^{Z Z}, \mu_{g g F}^{W W}$, and $\mu_{g g F}^{\tau \tau}$, while they are $\mu_{V B F}^{\gamma \gamma}, \mu_{V B F}^{W W}$, and $\mu_{V B F}^{\tau \tau}$ for $V B F$; these can be found from Table 3 of Ref. [29]. Additionally, we also consider the recent observation of $h \rightarrow b \bar{b}$ in $V h$ production by ATLAS [7] and CMS [8]. In order to determine the constraint on $\rho_{b b}$, we combine all these measurements and refer to them together as "Higgs signal strength measurements."

We now turn our attention to the $\mathcal{B}\left(B \rightarrow X_{s} \gamma\right)$ constraint. $\mathcal{B}\left(B \rightarrow X_{s} \gamma\right)$ receives contributions from charged Higgs and top quark loop, which modifies the leading order (LO) Wilson coefficient $C_{7,8}^{(0)}$ at the matching scale $\mu$. At the matching scale $\mu=m_{W}$ the LO Wilson coefficients are defined as

$$
C_{7,8}^{(0)}\left(m_{W}\right)=F_{7,8}^{(1)}\left(x_{t}\right)+\delta C_{7,8}^{(0)}\left(\mu_{W}\right)
$$

where $x_{t}=\left(\bar{m}_{t}\left(m_{W}\right) / m_{W}\right)^{2}, \bar{m}_{t}\left(m_{W}\right) \overline{\mathrm{MS}}$ running mass of top at $m_{W}$, and $F_{7,8}^{(1)}(x)$ can be found in Ref. [33] (see also Ref. [34]). The second term in Eq. (16) arises from the charged Higgs contribution, which is, at LO, expressed as [35]

$$
\delta C_{7,8}^{(0)}\left(m_{W}\right) \simeq \frac{\left|\rho_{t t}\right|^{2}}{3 \lambda_{t}^{2}} F_{7,8}^{(1)}\left(y_{H^{+}}\right)-\frac{\rho_{t t} \rho_{b b}}{\lambda_{t} \lambda_{b}} F_{7,8}^{(2)}\left(y_{H^{+}}\right),
$$

with $y_{H^{+}}=\left(\bar{m}_{t}\left(m_{W}\right) / m_{H^{+}}\right)^{2}$, while the expression for $F_{7,8}^{(2)}\left(y_{H^{+}}\right)$is given in Ref. [33]. In order to find the constraint on $\rho_{b b}$, we follow the prescription of Ref. [36] and define

$$
R_{\exp }=\frac{\mathcal{B}\left(B \rightarrow X_{s} \gamma\right)_{\exp }}{\mathcal{B}\left(B \rightarrow X_{s} \gamma\right)_{\mathrm{SM}}} .
$$

The current world average of $\mathcal{B}\left(B \rightarrow X_{s} \gamma\right)_{\text {exp }}$ extrapolated to photon energy cut $E_{0}=1.6 \mathrm{GeV}$ is $(3.32 \pm 0.15) \times 10^{-4}$ [37], while the next-to-next-to-LO prediction in SM for the same photon energy cut is $\mathcal{B}\left(B \rightarrow X_{s} \gamma\right)_{\mathrm{SM}}=(3.36 \pm 0.23) \times$ $10^{-4}$ [38]. We then demand $R_{\text {theory }}=\mathcal{B}\left(B \rightarrow X_{s} \gamma\right)_{\mathrm{G} 2 \mathrm{HDM}} /$ $\mathcal{B}\left(B \rightarrow X_{s} \gamma\right)_{\mathrm{SM}}$ based on our LO calculation. We take the matching scale and low-energy scale as $m_{W}$ and $\bar{m}_{b}\left(m_{b}\right)$, respectively, and demand $R_{\text {theory }}$ does not exceed $2 \sigma$ error of $R_{\text {exp }}$.

Recently, ACME Collaboration put a new constraint on electron $\operatorname{EDM}\left(d_{e}\right),\left|d_{e}\right|<1.1 \times 10^{-29} e \mathrm{~cm}$ [39], which is the most sensitive constraint on $\operatorname{Im}\left(\rho_{b b}\right){ }^{3}$ As widely studied, the two-loop Barr-Zee diagrams [44] are the leading contributions to $d_{e}$ in the 2HDM [45]. It is found that our $\rho_{b b}$-EWBG scenario would virtually be excluded by the new $d_{e}$ bound unless the cancellation mechanism or the alignment limit are invoked [16]. In the former case, for example, one gets $\left|d_{e}\right|=1.8 \times 10^{-29} e \mathrm{~cm}$ for $\operatorname{Im} \rho_{b b}=0.1$. This can be made smaller than the current experimental upper bound by turning on $\rho_{e e}$ as $\operatorname{Re} \rho_{e e}=0$ and $0.06 \lesssim \operatorname{Im} \rho_{e e} /\left(\lambda_{e} \lambda_{b}\right) \lesssim 0.3$ that induce other Barr-Zee diagrams with the opposite sign. In the latter case, all the EDM contributions are simply decoupled. In what follows, we assume the former in which phenomenological consequences are rich.

The direct $C P$ asymmetry $\mathcal{A}_{C P}$ [46] of $B \rightarrow X_{s} \gamma$ also offers a very sensitive probe for $\operatorname{Im}\left(\rho_{b b}\right)$. However, it has been proposed [47] that $\Delta \mathcal{A}_{\mathrm{CP}}$, i.e., the asymmetry of the $C P$ asymmetry for the charged and neutral $B \rightarrow X_{s} \gamma$ decay is even more powerful for probing $C P$ violating effects. $\Delta \mathcal{A}_{\mathrm{CP}}$ is defined as [47]

$\Delta \mathcal{A}_{\mathrm{CP}}=\mathcal{A}_{B^{-} \rightarrow X_{s}^{-} \gamma}-\mathcal{A}_{B^{0} \rightarrow X_{s}^{0} \gamma} \approx 4 \pi^{2} \alpha_{s} \frac{\tilde{\Lambda}_{78}}{m_{b}} \operatorname{Im}\left(\frac{C_{8}}{C_{7}}\right)$,

where $\tilde{\Lambda}_{78}$ is a hadronic parameter and $\alpha_{s}$ is the strong coupling constant at $\bar{m}_{b}\left(m_{b}\right)$. Recently, a Belle experiment reported that $\Delta \mathcal{A}_{\mathrm{CP}}=(+3.69 \pm 2.65 \pm 0.76) \% \quad[48],{ }^{4}$ where the first uncertainty is statistical and the second one is systematic. In order to find the excluded region for $\rho_{b b}$, we utilize Eq. (19), and allow a $2 \sigma$ error on the measured value of $\Delta \mathcal{A}_{\mathrm{CP}}$. In finding the constraint, we have utilized the LO Wilson coefficients as in Eq. (16) as first approximation. The hadronic parameter $\tilde{\Lambda}_{78}$ is expected to be $\sim \Lambda_{\mathrm{QCD}}$ and estimated to be in the range of $17 \mathrm{MeV}<$ $\tilde{\Lambda}_{78}<190 \mathrm{MeV}$ [47]. In our analysis we take the average value of $\tilde{\Lambda}_{78}=89 \mathrm{MeV}$ as a reference value. We remark that this constraint heavily depends on the value of $\tilde{\Lambda}_{78}$ and becomes weaker for the smaller values of $\tilde{\Lambda}_{78}$.

\section{RESULTS}

For illustration we set $c_{\beta-\alpha}=0.1$ and assume that $m_{H}=m_{A}=m_{H^{ \pm}}=600 \mathrm{GeV}$; however, the impact of

\footnotetext{
${ }^{3}$ We have confirmed that neutron and Mercury EDMs in our scenario are smaller than the current experimental bounds [40,41] by two- and one-order magnitudes, respectively, where the estimates are based on Refs. [42,43]. Note that a cancellation scenario described below does not change this situation.

${ }^{4}$ We are grateful to Akimasa Ishikawa for pointing out the changed central value and errors of $\Delta \mathcal{A}_{\mathrm{CP}}$ in the latest version of Ref. [48].
} 

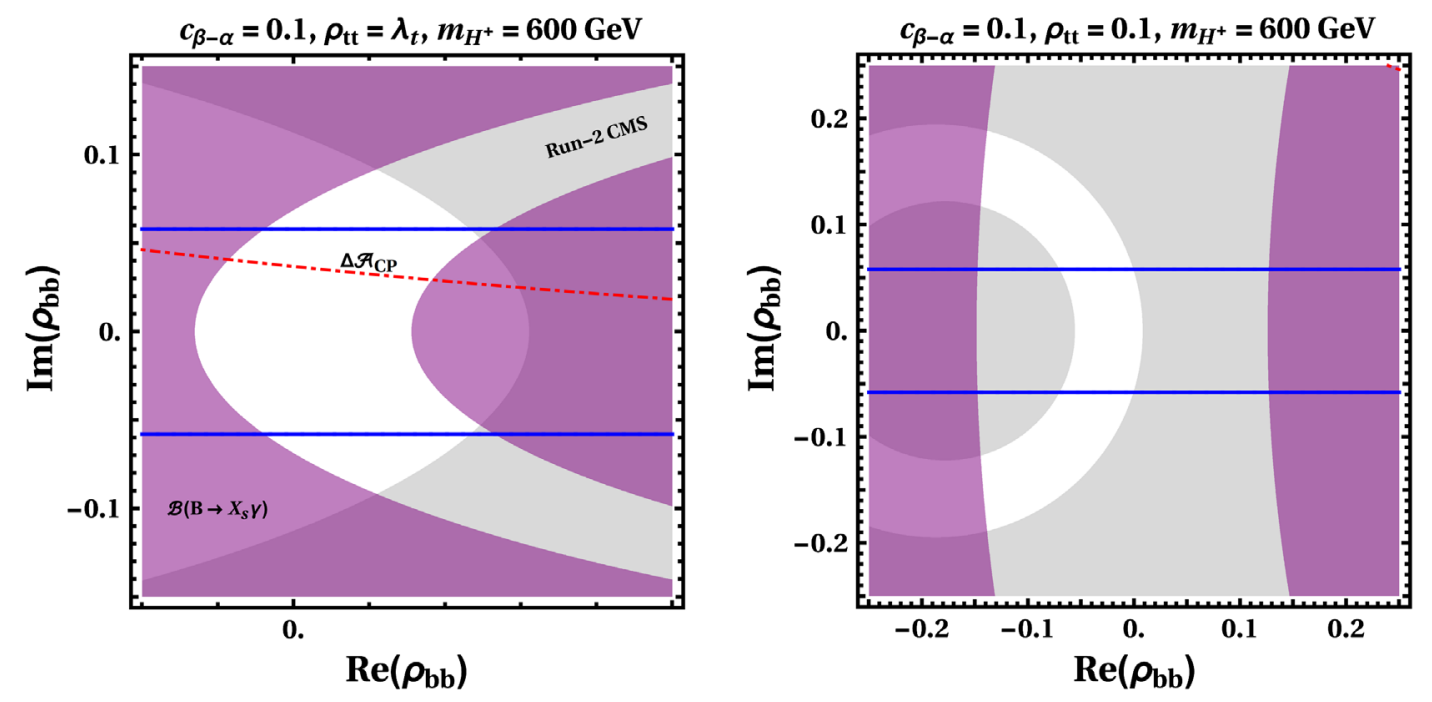

FIG. 1. $Y_{B} / Y_{B}^{\text {obs }}=1$ contours (blue solid contours) and the $2 \sigma$ excluded limits of the Higgs signal strengths (gray shaded regions), $\mathcal{B}\left(B \rightarrow X_{s} \gamma\right)$ (purple shaded regions), and $\Delta \mathcal{A}_{\mathrm{CP}}$ (red dash-dotted curves) are shown, respectively. We take $c_{\beta-\alpha}=0.1$, $m_{H}=m_{A}=m_{H^{ \pm}}=600 \mathrm{GeV}, \rho_{t t}=\lambda_{t}$ (left), and $\rho_{t t}=0.1$ (right).

other choices will be discussed in a later part of this paper. Furthermore, we take $\tan \beta=1$ and $M=400 \mathrm{GeV}$, where $M$ is a mixing mass parameter of the two Higgs doublet in a generic basis. This parameter choice corresponds to $\lambda_{1}=4.7, \lambda_{2}=2.4, \lambda_{3}=3.7, \lambda_{4}=\lambda_{5}=-3.3$, and $\lambda_{6}=$ $\lambda_{7}=0$ with the notation of Ref. [15]. ${ }^{5}$ With this choice, we have $T_{C}=112.4$ and $v\left(T_{C}\right)=191.3 \mathrm{GeV}$. For the input parameters for the $Y_{B}$ calculation, we take the parameters employed in Refs. $[15,16]$. One comment we should make here is that $Y_{B}$ is linearly proportional to $\Delta \beta$. Since its numerical value is unknown in the current model, we infer it from the results in the minimal supersymmetric standard model, i.e., $\Delta \beta=\mathcal{O}\left(10^{-4}-10^{-2}\right)$ [50]. Note that $\Delta \beta$ tends to be suppressed in the SM-like limit, which is realized by the large $m_{A}$ limit. In the 2HDM, however, the SM-like limits are controlled by both the heavy Higgs spectrum and $c_{\beta-\alpha}$. Since we do not take the exact alignment limit $\left(c_{\beta-\alpha}=0\right), \Delta \beta$ would not be so suppressed compared to the minimal supersymmetric standard model case with the same value of $m_{A}$. With this consideration, we take $|\Delta \beta|=$ 0.015 as a reference value.

In Fig. 1, the BAU-viable regions are shown with the current experimental constraints discussed above. We take $\rho_{t t}=\lambda_{t}$ (left panel) and 0.1 (right panel), respectively. The regions of $\left|\operatorname{Im}\left(\rho_{b b}\right)\right| \gtrsim 0.058$ give $Y_{B} / Y_{B}^{\text {obs }}>1$, which are indicated by the blue solid contours. Note that the regions of $\operatorname{Re}\left(\rho_{b b}\right) \gtrless 0$ and $\operatorname{Im}\left(\rho_{b b}\right) \gtrless 0$ correspond to $\Delta \beta \gtrless 0$, respectively. The shaded regions in gray (purple) are ruled out by the Higgs signal strength measurements

\footnotetext{
${ }^{5}$ It is found that $\lambda_{1}(Q)>4 \pi$ at $Q=2.7 \mathrm{TeV}$ and the Landau pole occurs at $Q=7.4 \mathrm{TeV}$ using one-loop renormalization equations. Such a low cutoff is a generic consequence of the strong first-order EWPT in 2HDMs [12,27,49].
}

$\left[\mathcal{B}\left(B \rightarrow X_{s} \gamma\right)\right]$ at the $2 \sigma$ level, while the $2 \sigma$ exclusion limits of $\Delta \mathcal{A}_{\mathrm{CP}}$ are indicated by the red dash-dotted curves (with the regions above the dash-dotted curve excluded). In our analysis, we symmetrized the errors in the Higgs signal strength measurements for simplicity. One can see that the EWBG-viable regions are rather limited by these current experimental constraints. For $\rho_{t t}=\lambda_{t}$, the regions conforming $\operatorname{Im}\left(\rho_{b b}\right) \gtrsim 0.058$ are excluded by $\Delta \mathcal{A}_{\mathrm{CP}}$ measurement [Fig. 1 (left)]; however, negative $\operatorname{Im}\left(\rho_{b b}\right)$ can still sustain $Y_{B} / Y_{B}^{\text {obs }}>1$, but $\left|\operatorname{Im}\left(\rho_{b b}\right)\right|$ cannot be $\gtrsim 0.1$. Note that in Fig. 1 (left), the $\Delta \mathcal{A}_{\mathrm{CP}}$ constraint excludes the EWBG-viable regions for $\operatorname{Im}\left(\rho_{b b}\right)>0$. This is because of the nonzero and positive central values of the Belle $\Delta \mathcal{A}_{\mathrm{CP}}$ measurement [48] and our choice of real and positive $\rho_{t t}=$ $\lambda_{t}$ in the left panel of Fig. 1. For example, if one chooses $\rho_{t t}=-\lambda_{t}$, the $\Delta \mathcal{A}_{\mathrm{CP}}$ constraint would exclude EWBGviable regions for $\operatorname{Im}\left(\rho_{b b}\right)<0$, however, would allow the parameter space for $\operatorname{Im}\left(\rho_{b b}\right)>0$. If $\rho_{t t}=0.1$, on the other hand, $\left|\operatorname{Im}\left(\rho_{b b}\right)\right|$ can reach around 0.2 and the EWBG-viable regions are expanded [Fig. 1 (right)]. Note that $\Delta \mathcal{A}_{\mathrm{CP}}$ does not give any useful bounds in this case. We note in passing that if we do not assume the cancellation mechanism for $d_{e}$, the current bound would exclude the regions of $\left|\operatorname{Im}\left(\rho_{b b}\right)\right| \gtrsim 0.06$, excluding the most EWBG-viable regions. We further remark that the current constraints in Fig. 1, heavily depend on $c_{\beta-\alpha}, \rho_{t t}$, and $m_{H^{ \pm}}$. For example, in the alignment limit, the constraint from Higgs signal strength measurements, i.e., the gray shaded region, would vanish. This is clear from the expression of $y_{h i j}^{f}$ [see Eq. (3)], where the terms proportional to $\rho_{i j}$ are modulated by $c_{\beta-\alpha}$. Moreover, $\mathcal{B}\left(B \rightarrow X_{s} \gamma\right)$ and $\Delta \mathcal{A}_{\mathrm{CP}}$ do not depend on $c_{\beta-\alpha}$, and the constraints from them will remain even for $c_{\beta-\alpha}=0$. However, these two constraints vanish if $\rho_{t t}=0$ and/or $m_{H^{ \pm}}$becomes too heavy. In such special case, i.e., 

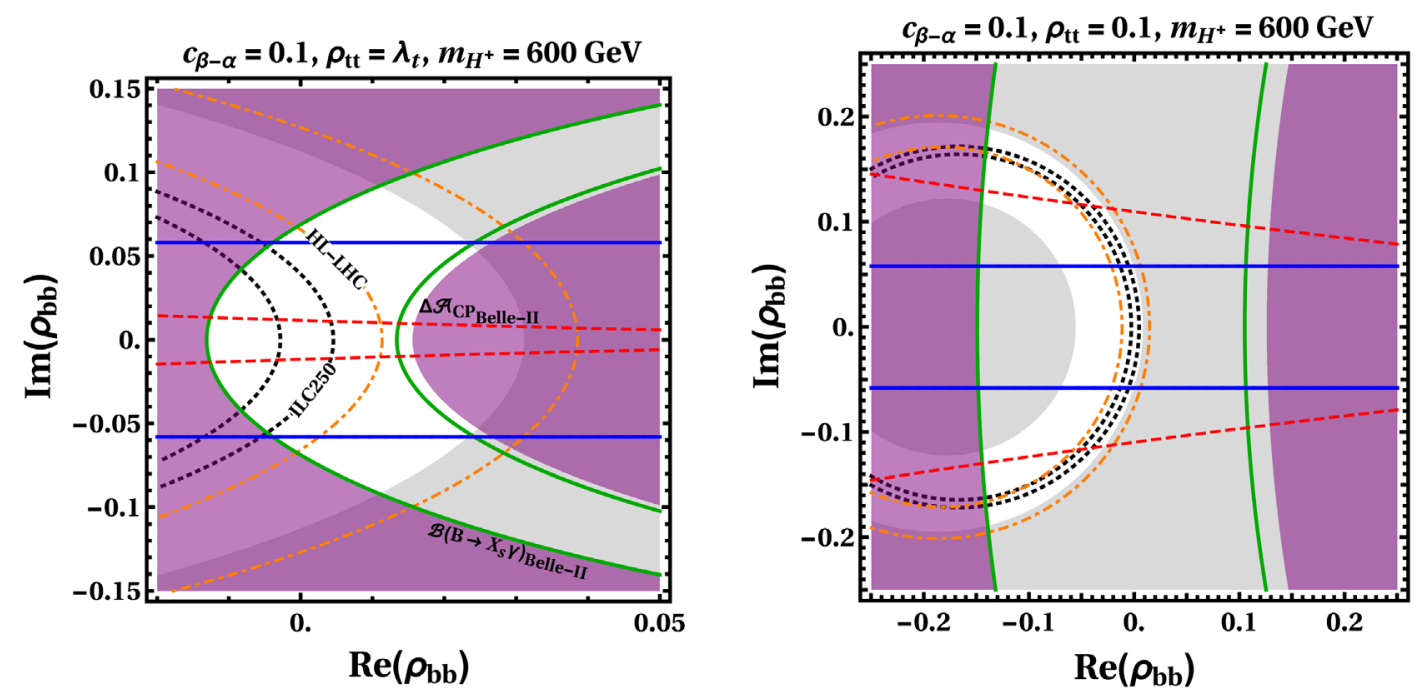

FIG. 2. Same as in Fig. 1 but future experimental sensitivities of HL-LHC (orange dash-dotted curves), ILC (black dotted curves), and Belle-II [green solid curves for $\mathcal{B}\left(B \rightarrow X_{s} \gamma\right)$ and red dotted curves for $\Delta \mathcal{A}_{\mathrm{CP}}$ ] are also overlaid. The central values for the future projection are assumed to be the same as in SM (Scenario 1).

when $\rho_{t t}=0$ and $c_{\beta-\alpha}=0$, the constraint on $\left|\operatorname{Im}\left(\rho_{b b}\right)\right|$ could be milder.

Now we discuss future prospects. The future measurements of these observables from Belle-II, full HL-LHC dataset $\left(3000 \mathrm{fb}^{-1}\right)$ will also provide a very sensitive probe. It will nonetheless be interesting to find out the parameter space for $\rho_{b b}$ assuming future projections of these constraints. In order to find the constraints from future projections, we adopt two different scenarios. In the first scenario (Scenario 1), we assume the central values of the future measurements for all these constraints are the same as in SM, while in the second scenario (Scenario 2) the central values are assumed to remain the same as in the current measurements. The parameter spaces for $\rho_{b b}$ with the projections in Scenario 1 are summarized in Fig. 2, while the projections with Scenario 2 are shown in Fig. 3.

Let us discuss the impact of these future projections in detail. The full HL-LHC dataset is expected to measure $\mu_{g g F}^{\gamma \gamma}$, $\mu_{g g F}^{Z Z}, \mu_{g g F}^{W W}, \mu_{V B F}^{\gamma \gamma}$, and $\mu_{V B F}^{W W}$ very precisely, leading to a very stringent constraint on $\rho_{b b}$. For example, with an integrated luminosity of $3000 \mathrm{fb}^{-1}$, the projected relative uncertainties by ATLAS and CMS [51,52] are $\sim 5 \%$ for $\mu_{g g F}^{\gamma \gamma}, \mu_{g g F}^{Z Z}, \mu_{g g F}^{W W}$, and $\sim 10 \%$ for $\mu_{V B F}^{\gamma \gamma}, \mu_{V B F}^{W W}$, respectively. We find the $2 \sigma$ orange dot-dashed contours in Figs. 2 and 3, assuming Scenarios 1 and 2, respectively. In addition to these limits, ILC could measure [53] the $h b b$ coupling at $1.1 \%(1 \sigma)$
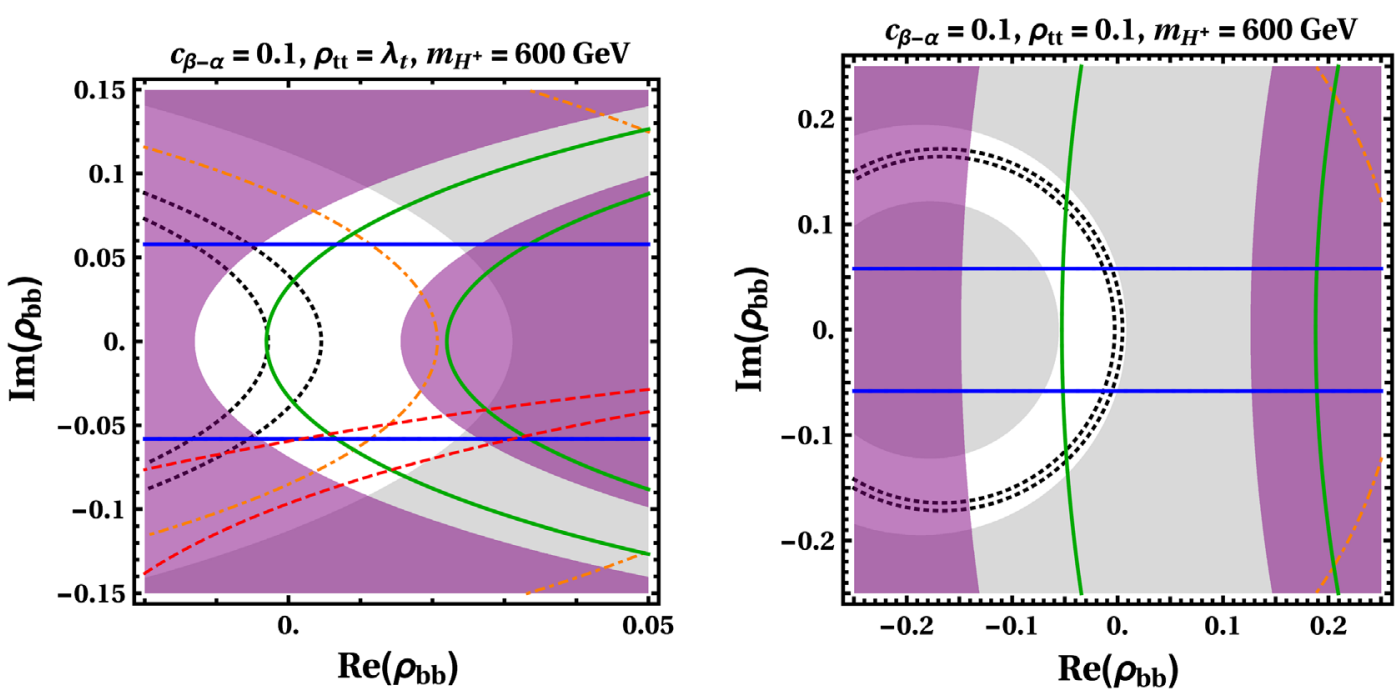

FIG. 3. Same as in Fig. 2 but the central values for the future projection is assumed to be the same as in the current measurements (Scenario 2). 
accuracy (relative to its SM value) in the $250 \mathrm{GeV}$ program ( $2 \mathrm{ab}^{-1}$ data). We show this projected limit ( $2 \sigma$ exclusion) by the black dotted contours in Figs. 2 and 3.

Belle-II will also provide a stringent constraint. The projected $2 \sigma$ exclusions from $\mathcal{B}\left(B \rightarrow X_{s} \gamma\right)$ are shown in Figs. 2 and 3 by green solid contours, while the projection for $\Delta \mathcal{A}_{\mathrm{CP}}$ is shown by red dashed contours. In finding these contours, we adopted a similar strategy as in the HL-LHC projection of the Higgs signal strength measurements and take two different scenarios for central values. For $\mathcal{B}\left(B \rightarrow X_{s} \gamma\right)$, we utilize the $3.2 \%$ relative uncertainty for Belle-II with $50 \mathrm{ab}^{-1}$ data [54] in our analysis. This projected uncertainty is for the leptonic tag $\mathcal{B}\left(B \rightarrow X_{s} \gamma\right)$ and is smaller than the hadronic tag or the combination of the both. On the other hand, the projected Belle-II $\left(50 \mathrm{ab}^{-1}\right)$ absolute uncertainty for $\Delta \mathcal{A}_{\mathrm{CP}}$ is $0.3 \%$ [54].

It is clear that the future measurements offer excellent tests for EWBG via bottom transport. These future measurements may indeed discover $\rho_{b b}$ driven EWBG. A discovery $(5 \sigma)$ would be intriguing. However, it would require larger $\rho_{b b}$ compared to the projected exclusion limits $(2 \sigma)$ of the different measurements as shown in Figs. 2 and 3. A large part of the EWBG-viable regions can be probed by these future measurements. For example, in Scenario 1, if $\rho_{t t}=\lambda_{t}$ (left panel of Fig. 2), constraints from HL-LHC (orange dot-dashed contours) and ILC-250 (black dotted contours) mutually exclude the regions required for $Y_{B} / Y_{B}^{\mathrm{obs}}>1$. Additionally, in this scenario, red dashed contours from future $\Delta \mathcal{A}_{\mathrm{CP}}$ measurements lie below $\left|\operatorname{Im}\left(\rho_{b b}\right)\right|=0.058$. However, if $\rho_{t t} \sim 0.1$, there exist regions where $\left|\operatorname{Im}\left(\rho_{b b}\right)\right| \gtrsim 0.058$. The situation becomes completely different for Scenario 2. In this scenario, HLLHC, ILC-250, and $\Delta \mathcal{A}_{\mathrm{CP}}$ mutually exclude all of the regions that can support $Y_{B} / Y_{B}^{\text {obs }}>1$ both for $\rho_{t t} \sim \lambda_{t}$ and $\rho_{t t} \sim 0.1$. This can easily be seen from Fig. 3. ${ }^{6}$ However, we stress again that the excluded regions from future projections depend on the assumptions made on the parameters while generating Figs. 2 and 3. As discussed earlier, the constraints from HL-LHC Higgs signal strength measurements and ILC-250 vanish if $c_{\beta-\alpha}=0$. Besides, the constraints from $\mathcal{B}\left(B \rightarrow X_{s} \gamma\right)$ and $\Delta \mathcal{A}_{\mathrm{CP}}$ would also vanish if $\rho_{t t}=0$ and $m_{H^{ \pm}}$becomes heavy. In such scenarios, there exist finite parameter spaces for $\left|\operatorname{Im}\left(\rho_{b b}\right)\right|$ to sustain $Y_{B} / Y_{B}^{\mathrm{obs}}>1$.

\section{DISCUSSIONS AND CONCLUSION}

The interpretation of the EWBG-viable regions needs some caution. As discussed in Ref. [15], the BAU is subject

\footnotetext{
${ }^{6}$ Note that the other orange contour lies in the right-hand side of the existing orange contour beyond the range shown in Fig. 3 (right). Besides, the red dashed contours for the future $\Delta \mathcal{A}_{\mathrm{CP}}$ measurement lie far below $\operatorname{Im}\left(\rho_{b b}\right)=0$. Hence, HL-LHC, ILC250 , and future $\Delta \mathcal{A}_{\mathrm{CP}}$ mutually exclude the entire BAU-viable regions in Fig. 3 (right)
}

to significant theoretical uncertainties (see also Ref. [4]). For example, we make use of the VEV insertion approximation that may lead to the overestimated BAU. Likewise, as mentioned above, ignorance of the $C P$-conserving term induced by the $\left(Y_{1,2}\right)_{b s}$ could also yield the overestimated BAU. In addition to those computational issues, the impreciseness of the input parameters are also the source of the theoretical uncertainties. In particular, if $\Delta \beta$ is found to be 1 order of magnitude smaller than the value we take here, the BAU would get smaller by 1 order ofmagnitude, eliminating the EWBG-viable regions. Furthermore, there exists a discrepancy between the $C P$-violating source term calculated by our method and the one by semiclassical force [12] (see also Ref. [25]). Since the former is first order in derivative while the latter is second in derivative, the BAU obtained by the latter scheme would become lower. Therefore, improvement of the BAU calculation is crucially important for the test of the scenario. If $\rho_{b b}$ turns out to be deficient to drive the sufficient BAU in a more refined calculation, the $\rho_{t t} / \rho_{t c}$ EWBG discussed in Ref. [16] would be the unique mechanism for baryogenesis in the G2HDM by virtue of its wider viable parameter space. Nonetheless, the definitive conclusion cannot be made until the refined BAU calculation is available.

The constraints from $\mathcal{B}\left(B \rightarrow X_{s} \gamma\right)$ and $\Delta \mathcal{A}_{\mathrm{CP}}$ measurements can probe a significant portion of the EWBG-viable parameter space. The $\Delta \mathcal{A}_{\mathrm{CP}}$ measurement with the full Belle-II $50 \mathrm{ab}^{-1}$ dataset can probe $\left|\operatorname{Im}\left(\rho_{b b}\right)\right| \gtrsim 0.1$ in Scenario 1 or even can rule out the entire BAU-viable region completely in Scenario 2, even for $\rho_{t t} \sim 0.1$. Although our assumptions on the central values for future measurements (i.e., Scenario 1 and Scenario 2) are very indicative, however, we stress that the program should be revisited after the actual future measurements. The recent measurements of $\mathcal{A}_{\mathrm{CP}}$ and isospin violating asymmetry $\left(\Delta_{0+}\right)$ of $B \rightarrow K^{*} \gamma$ decay by Belle [55] may also provide a complementary probe for $\operatorname{Im}\left(\rho_{b b}\right)$, although the theoretical predictions of these observables in general suffer from sizable uncertainties [56].

EDM probes could come into play if their measurements are significantly improved or newly available. For example, proton EDM, which is expected to reach $\sim 10^{-29} e \mathrm{~cm}$ at Brookhaven National Laboratory [57], could give a good opportunity to confirm our scenario since our prediction is around $10^{-28} \mathrm{ecm}$. Follow-up studies along this line are worth pursuing.

The future updates from HFLAV for the global average of $\mathcal{B}\left(B \rightarrow X_{s} \gamma\right)$ would also play a major role in constraining the BAU-viable region, if $\rho_{t t}$ is not vanishingly small. In this regard, we remark that the $B_{q}-\bar{B}_{q}(q=d, s)$ mixing [35] and the recent discovery of $t \bar{t} h[58,59]$ would provide independent probes [32] for $\rho_{t t}$.

The Higgs signal strength measurements at HL-LHC would be complementary in probing $\rho_{b b}$ regardless of the value of $\rho_{t t}$; however, $c_{\beta-\alpha}$ should not be very small. It should 
be noted that $\left|\operatorname{Im}\left(\rho_{b b}\right)\right|$ cannot be too large for nonzero $c_{\beta-\alpha}$. The current limit on the $h$ boson total width $\Gamma_{h}<0.013 \mathrm{GeV}$ (95\% C.L.) [1] sets the upper limit on $\left|\rho_{b b}\right|$ if $c_{\beta-\alpha} \neq 0$. Utilizing this limit we find that for $\operatorname{Re}\left(\rho_{b b}\right)=0$ and $c_{\beta-\alpha}=0.1,\left|\operatorname{Im}\left(\rho_{b b}\right)\right| \lesssim 0.36$ at $95 \%$ C.L. In determining the upper limit on $\left|\operatorname{Im}\left(\rho_{b b}\right)\right|$ we used the LO decay width of $h$ for simplicity. We also remark that, as the Run 1 combination [60], a Run 2 combined fit of ATLAS and CMS Higgs signal strengths would be more indicative. Further, our study illustrates that the ILC $250 \mathrm{GeV}$ run might probe $\rho_{b b}$ better than HL-LHC. It is not surprising that ILC, even its $250 \mathrm{GeV}$ program, presents a better probe for NP in bottom Yukawa than HL-LHC.

Also, LHC might offer direct detection of $\rho_{b b}$ driven EWBG. A nonzero $\operatorname{Im}\left(\rho_{b b}\right)$ induces the $g g \rightarrow b \bar{b} A(H) \rightarrow$ $b \bar{b} Z H(A)$ process if $m_{A}>m_{H}+m_{Z}\left(m_{H}>m_{A}+m_{Z}\right)$. This process provides a unique probe for the EWBG, even for $c_{\beta-\alpha}=0$ and/or $\rho_{t t}=0$. Notwithstanding, if $c_{\beta-\alpha}$ is not too small, a direct detection program can cover $g g \rightarrow b \bar{b} A \rightarrow b \bar{b} Z h$. A discovery would be intriguing. Furthermore, for moderate values of $\rho_{t t}, g g \rightarrow t \bar{t} A / t \bar{t} H \rightarrow$ $t \bar{t} b \bar{b}$ with leptonic decays of at least one top, and $A / H \rightarrow$ $b \bar{b}$ could be interesting. These would be studied elsewhere.

In conclusion, motivated by the recent discovery of Higgs boson decay to bottom quarks, we have analyzed the possibility of EWBG by extra bottom Yukawa $\rho_{b b}$ in the G2HDM. After satisfying all existing constraints, we found that indeed $\rho_{b b}$ can generate successful BAU; however, $\left|\operatorname{Im}\left(\rho_{b b}\right)\right|$ is required to be $\gtrsim 0.058$. For a wide range of parameter space, future measurements from Belle-II, Higgs signal strengths at HL-LHC, and ILC will provide exquisite probes for such a scenario. If the additional scalar and pseudoscalar are in the sub-TeV range, the program can also be covered by direct searches at LHC.

\section{ACKNOWLEDGMENTS}

We thank Wei-Shu Hou and Masaya Kohda for discussions. We also thank Akimasa Ishikawa for communication and discussions. T. M. is supported by Grant No. MOST-107-2811-M-002-3069 of Taiwan, Republic of China, and E. S. is supported by IBS under Project Code IBS-R018-D1.
[1] M. Tanabashi et al. (Particle Data Group), Phys. Rev. D 98, 030001 (2018).

[2] G. Aad et al. (ATLAS Collaboration), Phys. Lett. B 716, 1 (2012); S. Chatrchyan et al. (CMS Collaboration), Phys. Lett. B 716, 30 (2012).

[3] V. A. Kuzmin, V. A. Rubakov, and M. E. Shaposhnikov, Phys. Lett. B 155, 36 (1985); For some reviews, see e.g., M. Quiros, Helv. Phys. Acta 67, 451 (1994); V. A. Rubakov and M. E. Shaposhnikov, Usp. Fiz. Nauk 166, 493 (1996) [Phys. Usp. 39, 461 (1996)]; K. Funakubo, Prog. Theor. Phys. 96, 475 (1996); A. Riotto, arXiv:hep-ph/9807454; W. Bernreuther, Lect. Notes Phys. 591, 237 (2002); J. M. Cline, arXiv:hep-ph/0609145; T. Konstandin, Phys. Usp. 56, 747 (2013).

[4] D. E. Morrissey and M. J. Ramsey-Musolf, New J. Phys. 14, 125003 (2012).

[5] M. Carena, A. Megevand, M. Quiros, and C. E. M. Wagner, Nucl. Phys. B716, 319 (2005).

[6] P. Huang, A. J. Long, and L. T. Wang, Phys. Rev. D 94, 075008 (2016).

[7] M. Aaboud et al. (ATLAS Collaboration), Phys. Lett. B 786, 59 (2018).

[8] A. M. Sirunyan et al. (CMS Collaboration), Phys. Rev. Lett. 121, 121801 (2018).

[9] N. Cabibbo, Phys. Rev. Lett. 10, 531 (1963); M. Kobayashi and T. Maskawa, Prog. Theor. Phys. 49, 652 (1973).

[10] G. C. Branco, P. M. Ferreira, L. Lavoura, M. N. Rebelo, M. Sher, and J. P. Silva, Phys. Rep. 516, 1 (2012).

[11] S. Tulin and P. Winslow, Phys. Rev. D 84, 034013 (2011).
[12] J. M. Cline, K. Kainulainen, and M. Trott, J. High Energy Phys. 11 (2011) 089.

[13] H. K. Guo, Y. Y. Li, T. Liu, M. Ramsey-Musolf, and J. Shu, Phys. Rev. D 96, 115034 (2017).

[14] T. Liu, M. J. Ramsey-Musolf, and J. Shu, Phys. Rev. Lett. 108, 221301 (2012).

[15] C. W. Chiang, K. Fuyuto, and E. Senaha, Phys. Lett. B 762 , 315 (2016).

[16] K. Fuyuto, W. S. Hou, and E. Senaha, Phys. Lett. B 776, 402 (2018).

[17] J. de Vries, M. Postma, J. van de Vis, and G. White, J. High Energy Phys. 01 (2018) 089.

[18] A. Riotto, Nucl. Phys. B518, 339 (1998); Phys. Rev. D 58, 095009 (1998).

[19] C. Lee, V. Cirigliano, and M. J. Ramsey-Musolf, Phys. Rev. D 71, 075010 (2005); V. Cirigliano, M. J. Ramsey-Musolf, S. Tulin, and C. Lee, Phys. Rev. D 73, 115009 (2006); D. J. H. Chung, B. Garbrecht, M. J. Ramsey-Musolf, and S. Tulin, J. High Energy Phys. 12 (2009) 067; D. J. H. Chung, B. Garbrecht, M. J. Ramsey-Musolf, and S. Tulin, Phys. Rev. D 81, 063506 (2010).

[20] M. Carena, J. M. Moreno, M. Quiros, M. Seco, and C. E. M. Wagner, Nucl. Phys. B599, 158 (2001); M. Carena, M. Quiros, M. Seco, and C. E. M. Wagner, Nucl. Phys. B650, 24 (2003).

[21] T. Prokopec, M. G. Schmidt, and S. Weinstock, Ann. Phys. (Berlin) 314, 208 (2004); 314, 267 (2004); T. Konstandin, T. Prokopec, and M. G. Schmidt, Nucl. Phys. B716, 373 (2005); T. Konstandin, T. Prokopec, M. G. Schmidt, and M. Seco, Nucl. Phys. B738, 1 (2006). 
[22] V. Cirigliano, C. Lee, M. J. Ramsey-Musolf, and S. Tulin, Phys. Rev. D 81, 103503 (2010); V. Cirigliano, C. Lee, and S. Tulin, Phys. Rev. D 84, 056006 (2011).

[23] R. N. Mohapatra and X. m. Zhang, Phys. Rev. D 45, 2699 (1992); G. F. Giudice and M. E. Shaposhnikov, Phys. Lett. B 326, 118 (1994).

[24] P. Huet and A. E. Nelson, Phys. Rev. D 53, 4578 (1996).

[25] J. M. Cline, M. Joyce, and K. Kainulainen, J. High Energy Phys. 07 (2000) 018; L. Fromme, S. J. Huber, and M. Seniuch, J. High Energy Phys. 11 (2006) 038; L. Fromme and S. J. Huber, J. High Energy Phys. 03 (2007) 049.

[26] M. Ahmadvand, Int. J. Mod. Phys. A 29, 1450090 (2014).

[27] G. C. Dorsch, S. J. Huber, T. Konstandin, and J. M. No, J. Cosmol. Astropart. Phys. 05 (2017) 052.

[28] P. A. R. Ade et al. (Planck Collaboration), Astron. Astrophys. 571, A16 (2014).

[29] A. M. Sirunyan et al. (CMS Collaboration), Eur. Phys. J. C 79, 421 (2019).

[30] A. Djouadi, Phys. Rep. 457, 1 (2008).

[31] D. Fontes, J. C. Romo, and J. P. Silva, J. High Energy Phys. 12 (2014) 043.

[32] W. S. Hou, M. Kohda, and T. Modak, Phys. Rev. D 98, 075007 (2018).

[33] M. Ciuchini, G. Degrassi, P. Gambino, and G. F. Giudice, Nucl. Phys. B527, 21 (1998).

[34] K. G. Chetyrkin, M. Misiak, and M. Munz, Phys. Lett. B 400, 206 (1997); 425, 414(E) (1998).

[35] B. Altunkaynak, W. S. Hou, C. Kao, M. Kohda, and B. McCoy, Phys. Lett. B 751, 135 (2015).

[36] A. Crivellin, A. Kokulu, and C. Greub, Phys. Rev. D 87, 094031 (2013).

[37] Y. Amhis et al. (HFLAV Collaboration), Eur. Phys. J. C 77, 895 (2017).

[38] M. Czakon, P. Fiedler, T. Huber, M. Misiak, T. Schutzmeier, and M. Steinhauser, J. High Energy Phys. 04 (2015) 168.

[39] V. Andreev et al. (ACME Collaboration), Nature (London) 562, 355 (2018).

[40] C. A. Baker et al., Phys. Rev. Lett. 97, 131801 (2006).
[41] B. Graner, Y. Chen, E. G. Lindahl, and B. R. Heckel, Phys. Rev. Lett. 116, 161601 (2016); 119, 119901(E) (2017).

[42] J. Hisano, D. Kobayashi, W. Kuramoto, and T. Kuwahara, J. High Energy Phys. 11 (2015) 085.

[43] K. Cheung, J. S. Lee, E. Senaha, and P. Y. Tseng, J. High Energy Phys. 06 (2014) 149.

[44] S. M. Barr and A. Zee, Phys. Rev. Lett. 65, 21 (1990); 65, 2920(E) (1990).

[45] M. Jung and A. Pich, J. High Energy Phys. 04 (2014) 076; S. Inoue, M. J. Ramsey-Musolf, and Y. Zhang, Phys. Rev. D 89, 115023 (2014); K. Cheung, J. S. Lee, E. Senaha, and P. Y. Tseng, J. High Energy Phys. 06 (2014) 149; T. Abe, J. Hisano, T. Kitahara, and K. Tobioka, J. High Energy Phys. 01 (2014) 106; , 04 (2016) 161.

[46] A. L. Kagan and M. Neubert, Phys. Rev. D 58, 094012 (1998).

[47] M. Benzke, S. J. Lee, M. Neubert, and G. Paz, Phys. Rev. Lett. 106, 141801 (2011).

[48] S. Watanuki et al., Phys. Rev. D 99, 032012 (2019).

[49] K. Fuyuto and E. Senaha, Phys. Lett. B 747, 152 (2015).

[50] J. M. Moreno, M. Quiros, and M. Seco, Nucl. Phys. B526, 489 (1998).

[51] ATLAS Collaboration, Report No. ATL-PHYS-PUB-2014016.

[52] CMS Collaboration, Report No. CMS-PAS-FTR-16-002.

[53] K. Fujii et al., arXiv:1710.07621.

[54] E. Kou et al. (Belle II Collaboration), arXiv:1808.10567.

[55] T. Horiguchi et al. (Belle Collaboration), Phys. Rev. Lett. 119, 191802 (2017).

[56] T. Hurth and M. Nakao, Annu. Rev. Nucl. Part. Sci. 60, 645 (2010).

[57] V. Anastassopoulos et al., Rev. Sci. Instrum. 87, 115116 (2016).

[58] M. Aaboud et al. (ATLAS Collaboration), Phys. Lett. B 784, 173 (2018).

[59] A. M. Sirunyan et al. (CMS Collaboration), Phys. Rev. Lett. 120, 231801 (2018).

[60] G. Aad et al. (ATLAS and CMS Collaborations), J. High Energy Phys. 08 (2016) 045. 